\title{
CONSERVATIVE MOTION OF DISCRETE, HEXAHEDRAL GYROSCOPE
}

Donald Greenspan

TR \# 315 


\title{
CONSERVATIVE MOTION OF A DISCRETE, HEXAHEDRAL GYROSCOPE
}

\author{
Donald Greenspan
}

\section{ABSTRACT:}

Gyroscopic motion is simulated by applying a molecular dynamics formulation to a rigid hexahedron. The conservative dynamical differential equations are solved numerically in such a fashion that all the system invariants are preserved. Examples which included precession, nutation, and a combination of looping and cusp formation are described and discussed.

1. Introduction. Rigid body motion is of fundamental interest in mathematics, science, and engineering. In this paper we will study a simplistic approach to this area of study in the spirit of molecular mechanics. In particular, we will consider a discrete, hexahedral body whose six faces are congruent, regular triangles and simulate its motion as it spins like a gyroscope. The approach will not require the use of special coordinates, Cayley-Klein parameters, tensors, dyadics, or related concepts ${ }^{1}$. All that will be required is three dimensional XYZ space and Newtonian mechanics. The numerical methodology will conserve exactly the same energy, linear momentum, and angular momentum as does the associated differential system. Computer examples, run in double precision on a 64 bit Alpha 275 personal computer, will be described and discussed.

2. A Discrete, Rigid Hexahedral Gyroscope. As shown in Figure 1, consider a hexahedron with six congruent, regular triangular faces. Let the edge length of each triangle be $\mathrm{R}$ and let the vertices be $P_{i}\left(x_{i}, y_{i}, z_{i}\right), i=1,2,3,4,5$. Initially, let

$$
\begin{aligned}
&\left(x_{1}, y_{1}, z_{1}\right)=(0,0,0),\left(x_{2}, y_{2}, z_{2}\right)=(0, R \sqrt{3} / 3, R \sqrt{6} / 3) \\
&\left(x_{3}, y_{3}, z_{3}\right)=\left(\frac{1}{2} R,-R \sqrt{3} / 6, R \sqrt{6} / 3,\right.\left(x_{4}, y_{4}, z_{4}\right)=\left(-\frac{1}{2} R,-R \sqrt{3} / 6, R \sqrt{6} / 3\right) \\
&\left(x_{5}, y_{5}, z_{5}\right)=(0,0,2 R \sqrt{6} / 3)
\end{aligned}
$$

The geometric center $\bar{P}=(\bar{x}, \bar{y}, \bar{z})$ of the hexahedron is $(0,0, R \sqrt{6} / 3)$

We define the neighbors of $P_{1}$ and $P_{5}$ to be $P_{2}, P_{3}, P_{4}$. The neighbors of any one of $P_{2}, P_{3}, P_{4}$ are defined to be all the other vertices. thus, $P_{1}$ and $P_{5}$ have three neighbors each, while $P_{2}, P_{3}$ and $P_{4}$ have four.

Next, let us set $P_{2}, P_{3}, P_{4}$ in rotation in the plane $z=R \sqrt{6} / 3$. As shown in Figure 2, let the velocity of each particle be perpendicular to the line joining that particle to the geometric center $\bar{P}$. Assume that $P_{2}, P_{3}, P_{4}$ each has the same speed $V$, so that we take the initial velocities $\vec{v}_{i}=\left(v_{i x}, v_{i y}, v_{i z}\right), i=1,2,3,4,5$, of each $P_{i}$ to be

$$
\begin{gathered}
\vec{v}_{1}=(0,0,0), \vec{v}_{2}=(V, 0,0), \vec{v}_{3}=\left(-\frac{1}{2} V,-V \sqrt{3} / 2,0\right) \\
\vec{v}_{4}=\left(-\frac{1}{2} V, V \sqrt{3} / 2,0\right), \vec{v}_{5}=(0,0,0) .
\end{gathered}
$$




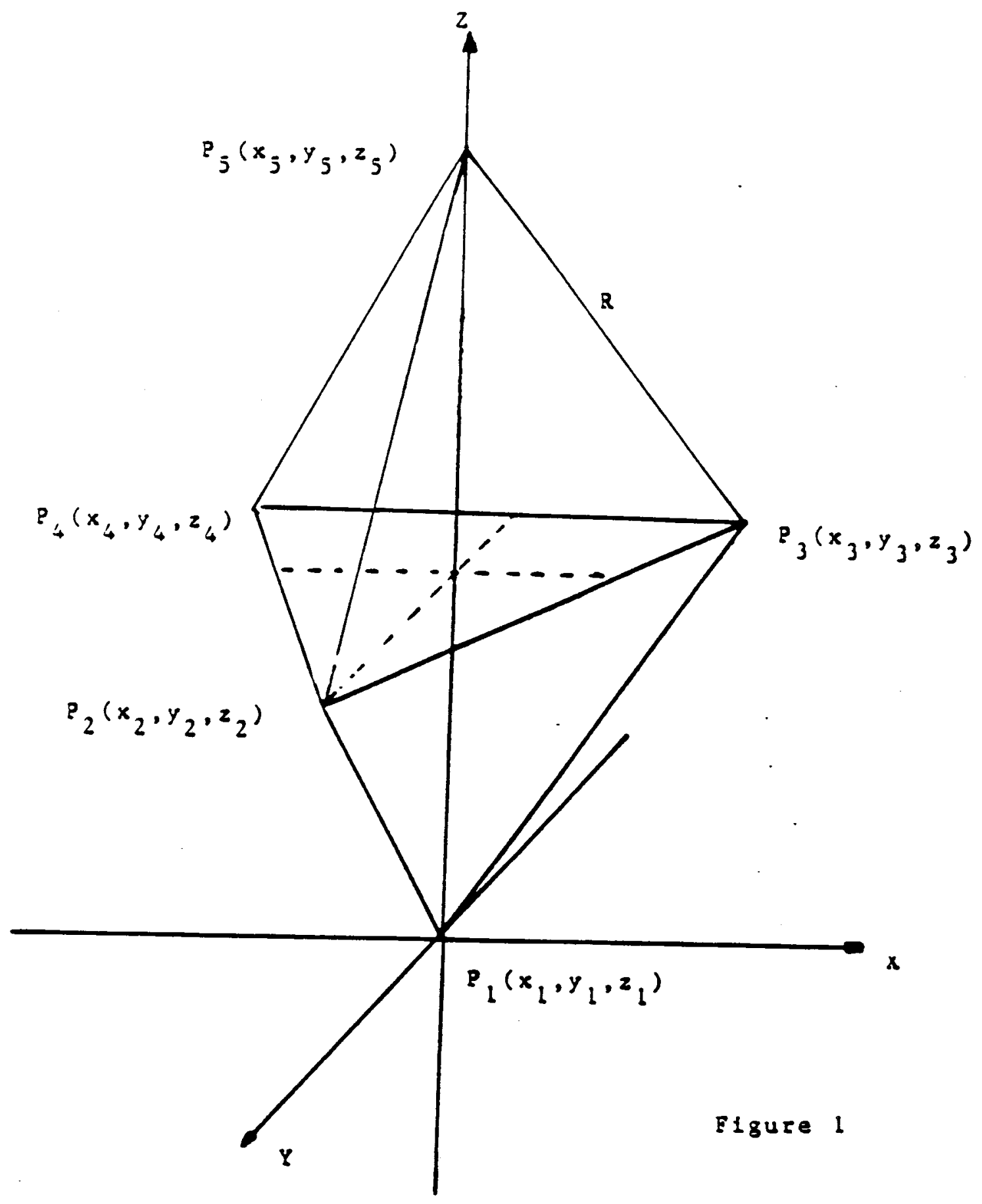


$\varepsilon$

$2 \operatorname{sn} 8 \mathrm{Ta}$

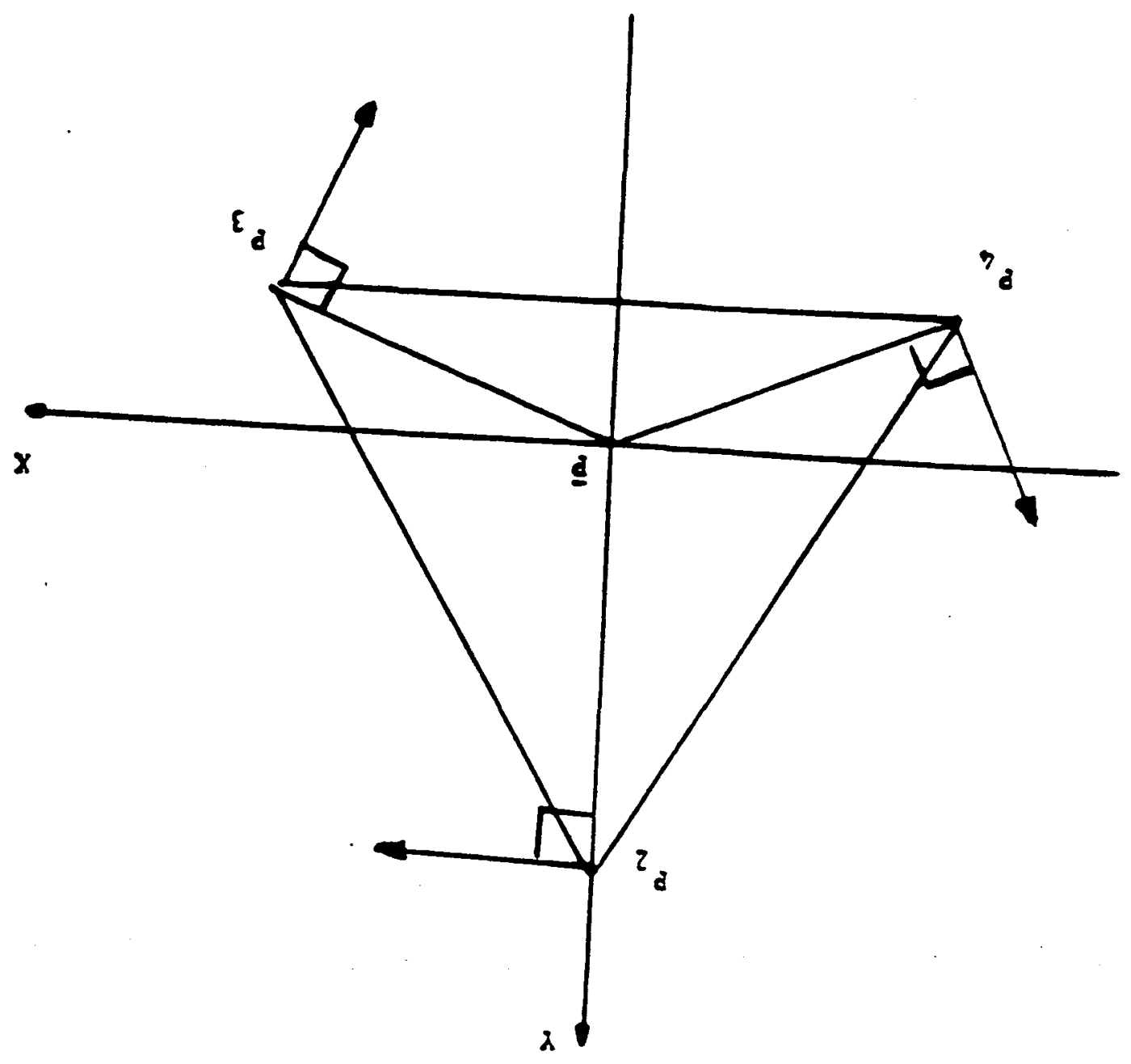


Finally, we want the rotating hexahedron to be tilted, initially, relative to the $Z$ axis, so that we assume that the line joining $P_{1}$ to the geometric center $\bar{P}$ forms an angle $\alpha$ relative to the $Z$ axis. As shown in Figure 3, this will be done by rotating the $X Z$ plane through an angle $\alpha$. Thus, the new positions $\left(x_{i}^{\prime}, y_{i}^{\prime}, z_{i}^{\prime}\right)$ and the new velocities $\left(v_{i x^{\prime}}\right.$, $\left.v_{i y^{\prime}}, v_{i z^{\prime}}\right), i=1,2,3,4,5$, satisfy

$$
\begin{gathered}
x_{i}^{\prime}=x_{i} \cos \alpha+z_{i} \sin \alpha, y_{i}^{\prime}=y_{i}, z_{i}^{\prime}=-x_{i} \sin \alpha+z_{i} \cos \alpha, \\
v_{i x^{\prime}}=v_{i x} \cos \alpha+v_{i z} \sin \alpha, v_{i y^{\prime}}=v_{i y}, v_{i z^{\prime}}=-v_{i x} \sin \alpha+v_{i z} \cos \alpha .
\end{gathered}
$$

Thus, once the parameters $R, V$, and $\alpha$ are given, all initial data for a tilted, rotating, hexahedral gyroscrope are determined.

3. Dynamical Equations. The motion of our rotating gyroscope is now treated as a five-body problem. At any time $t$, let $P_{i}, i=1,2,3,4,5$, be located at $\vec{r}_{i}=\left(x_{i}, y_{i}\right.$, $\left.z_{i}\right)$, have velocity $\vec{v}_{i}=\left(\dot{x}_{i}, \dot{y}_{i}, \dot{z}_{i}\right)=\left(v_{i x}, v_{i y}, v_{i z}\right)$, and have acceleration $\vec{a}_{i}=\left(\ddot{x}_{i}, \ddot{y}_{i}, \ddot{z}_{i}\right)=\left(\dot{v}_{i x}, \dot{v}_{i y}, \dot{v}_{i z}\right)$. Let the mass of each $P_{i}$ be $m_{i}$. For $i \neq j$, let $\vec{r}_{i j}$ be the vector from $P_{i}$ to $P_{j}$ and let $r_{i j}$ be the magnitude of $\vec{r}_{i j}, i=1,2,3,4,5$; $j=1,2,3,4,5 ; i \neq j$. Let $\phi=\phi\left(r_{i j}\right)$ be a potential function defined by the pair $P_{i}, P_{j}$, $i \neq j$. Then, for $i=1,2,3,4,5$, the Newtonian dynamical equations for the motion of the particles are the second order differential equations.

$$
\begin{aligned}
& m_{i} a_{i x}=-\frac{\partial \phi}{\partial r_{i j}} \frac{x_{i}-x_{j}}{r_{i j}}-\frac{\partial \phi}{\partial r_{i k}} \frac{x_{i}-x_{k}}{r_{i k}}-\frac{\partial \phi}{\partial r_{i m}} \frac{x_{i}-x_{m}}{r_{i m}}-\delta_{i} \frac{\partial \phi}{\partial r_{i n}} \frac{x_{i}-x_{n}}{r_{i n}} \\
& m_{i} a_{i y}=-\frac{\partial \phi}{\partial r_{i j}} \frac{y_{i}-y_{j}}{r_{i j}}-\frac{\partial \phi}{\partial r_{i k}} \frac{y_{i}-y_{k}}{r_{i k}}-\frac{\partial \phi}{\partial r_{i m}} \frac{y_{i}-y_{m}}{r_{i m}}-\delta_{i} \frac{\partial \phi}{\partial r_{i n}} \frac{y_{i}-y_{n}}{r_{i n}} \\
& m_{i} a_{i z}=-\frac{\partial \phi}{\partial r_{i j}} \frac{z_{i}-z_{j}}{r_{i j}}-\frac{\partial \phi}{\partial r_{i k}} \frac{z_{i}-z_{k}}{r_{i k}}-\frac{\partial \phi}{\partial r_{i m}} \frac{z_{i}-z_{m}}{r_{i m}}-\delta_{i} \frac{\partial \phi}{\partial r_{i n}} \frac{z_{i}-z_{n}}{r_{i n}}-g_{i} .
\end{aligned}
$$

In (3.1)-(3.3), $\delta_{1}=\delta_{5}=0, \delta_{2}=\delta_{3}=\delta_{4}=1$. Moreover, if $i=1$, then $j=2, k=3$, $m=4$; if $i=2$, then $j=1, k=3, m=4, n=5$; if $i=3$, then $j=1, k=2, m=4$, $n=5$; if $i=4$, then $j=1, k=2, m=3, n=5$; if $i=5$, then $j=2, k=3, m=4$. Moreover, we choose $g_{1}=0, g_{2}=g_{3}=g_{4}=g_{5}>0$, so that gravity acts only on $P_{2}$, $P_{3}, P_{4}, P_{5}$.

Equations (3.1)-(3.3) are fully conservative, that is, they conserve system energy, linear momentum and angular momentum, which are called the system invariants ${ }^{1}$.

4. Numerical Method. In order to solve system (3.1)-(3.3) in a fashion which conserves exactly the same energy, linear momentum and angular momentum, we first rewrite (3.1)-(3.3) as the following equivalent first order system: 


$$
\begin{aligned}
& \frac{d x_{i}}{d t}=v_{i x} \\
& \frac{d y_{i}}{d t}=v_{i y} \\
& \frac{d z_{i}}{d t}=v_{i z}
\end{aligned}
$$

$$
m_{i} \frac{d v_{i \pi}}{d t}=-\frac{\partial \phi}{\partial r_{i j}} \frac{x_{i}-x_{j}}{r_{i j}}-\frac{\partial \phi}{\partial r_{i k}} \frac{x_{i}-x_{k}}{r_{i k}}-\frac{\partial \phi}{\partial r_{i m}} \frac{x_{i}-x_{m}}{r_{i m}}-\delta_{i} \frac{\partial \phi}{\partial r_{i n}} \frac{x_{i}-x_{n}}{r_{i n}}
$$

$$
\begin{aligned}
& m_{i} \frac{d v_{i y}}{d t}=-\frac{\partial \phi}{\partial r_{i j}} \frac{y_{i}-y_{j}}{r_{i j}}-\frac{\partial \phi}{\partial r_{i k}} \frac{y_{i}-y_{k}}{r_{i k}}-\frac{\partial \phi}{\partial r_{i m}} \frac{y_{i}-y_{m}}{r_{i m}}-\delta_{i} \frac{\partial \phi}{\partial r_{i n}} \frac{y_{i}-y_{n}}{r_{i n}} \\
& m_{i} \frac{d v_{i z}}{d t}=-\frac{\partial \phi}{\partial r_{i j}} \frac{z_{i}-z_{j}}{r_{i j}}-\frac{\partial \phi}{\partial r_{i k}} \frac{z_{i}-z_{k}}{r_{i k}}-\frac{\partial \phi}{\partial r_{i m}} \frac{z_{i}-z_{m}}{r_{i m}}-\delta_{i} \frac{\partial \phi}{\partial r_{i n}} \frac{z_{i}-z_{n}}{r_{i n}}-g_{i} .
\end{aligned}
$$

We now choose difference equation approximations for (4.1)-(4.6). For a fixed time step $\Delta t$, let $t_{N}=N \Delta t, N=0,1,2,3, \ldots$. At $t_{N}$ let $P_{i}$ be at $\vec{r}_{i, N}=\left(x_{i, N}\right.$, $\left.y_{i, N}, z_{i, N}\right)$ with velocity $\vec{v}_{i, N}=\left(v_{i, z, N}, v_{i, y, N}, v_{i, z, N}\right)$. Then (4.1)-(4.6) will be approximated by

$$
\begin{aligned}
& \frac{x_{i, N+1}-x_{i, N}}{\Delta t}=\frac{v_{i, x, N+1}+v_{i, x, N}}{2} \\
& \frac{y_{i, N+1}-y_{i, N}}{\Delta t}=\frac{v_{i, y, N+1}+v_{i, \gamma, N}}{2} \\
& \frac{z_{i, N+1}-z_{i, N}}{\Delta t}=\frac{v_{i, 2, N+1}+v_{i, 2, N}}{2} \\
& m_{i} \frac{v_{i, 2, N+1}-v_{i, x, N}}{\Delta t}=-\frac{\phi\left(r_{i j, N+1}\right)-\phi\left(r_{i j, N}\right)}{r_{i j, N+1}-r_{i j, N}} \cdot \frac{x_{i, N+1}+x_{i, N}-x_{j, N+1}-x_{j, N}}{r_{i j, N+1}+r_{i j, N}} \\
& -\frac{\phi\left(r_{i k, N+1}\right)-\phi\left(r_{i k, N}\right)}{r_{i k, N+1}-r_{i k, N}} \cdot \frac{x_{i, N+1}+x_{i, N}-x_{k, N+1}-x_{k, N}}{r_{i k, N+1}+r_{i k, N}} \\
& -\frac{\phi\left(r_{i m, N+1}\right)-\phi\left(r_{i m, N}\right)}{r_{i m, N+1}-r_{i m, N}} \cdot \frac{x_{i, N+1}+x_{i, N}-x_{m, N+1}-x_{m_{3}, N}}{r_{i m, N+1}+r_{i m, N}} \\
& -\delta_{i} \frac{\phi\left(r_{i n, N+1}\right)-\phi\left(r_{i n, N}\right)}{r_{i n, N+1}-r_{i n, N}} \cdot \frac{x_{i, N+1}+x_{i, N}-x_{n, N+1}-x_{n, N}}{r_{i n, N+1}+r_{i n, N}}
\end{aligned}
$$

(4.11) $m_{i} \frac{v_{i, v, N+1}-v_{i, y, N}}{\Delta t}=-\frac{\phi\left(r_{i j, N+1}\right)-\phi\left(r_{i j, N}\right)}{r_{i j, N+1}-r_{i j, N}} \cdot \frac{y_{i, N+1}+y_{i, N}-y_{j, N+1}-y_{j, N}}{r_{i j, N+1}+r_{i j, N}}$

$$
-\frac{\phi\left(r_{i k, N+1}\right)-\phi\left(r_{i k, N}\right)}{r_{i k, N+1}-r_{i k, N}} \cdot \frac{y_{i, N+1}+y_{i, N}-y_{k, N+1}-y_{k, N}}{r_{i k, N+1}+r_{i k, N}}
$$




$$
\begin{aligned}
& -\frac{\phi\left(r_{i m, N+1}\right)-\phi\left(r_{i m, N}\right)}{r_{i m, N+1}-r_{i m, N}} \cdot \frac{y_{i, N+1}+y_{i, N}-y_{m, N+1}-y_{m, N}}{r_{i m, N+1}+r_{i m, N}} \\
& -\delta_{i} \frac{\phi\left(r_{i n, N+1}\right)-\phi\left(r_{i n, N}\right)}{r_{i n, N+1}-r_{i n, N}} \cdot \frac{y_{i, N+1}+y_{i, N}-y_{n, N+1}-y_{n, N}}{r_{i n, N+1}+r_{i n, N}}
\end{aligned}
$$

$$
\begin{aligned}
m_{i} \frac{v_{i, z, N+1}-v_{i, z, N}}{\Delta t}= & -\frac{\phi\left(r_{i j, N+1}\right)-\phi\left(r_{i j, N}\right)}{r_{i j, N+1}-r_{i j, N}} \cdot \frac{z_{i, N+1}+z_{i, N}-z_{j, N+1}-z_{j, N}}{r_{i j, N+1}+r_{i j, N}} \\
& -\frac{\phi\left(r_{i k, N+1}\right)-\phi\left(r_{i k, N}\right)}{r_{i k, N+1}-r_{i k, N}} \cdot \frac{z_{i, N+1}+z_{i, N}-z_{k, N+1}-z_{k, N}}{r_{i k, N+1}+r_{i k, N}} \\
& -\frac{\phi\left(r_{i m, N+1}\right)-\phi\left(r_{i m, N}\right)}{r_{i m, N+1}-r_{i m, N}} \cdot \frac{z_{i, N+1}+z_{i, N}-z_{m, N+1}-z_{m, N}}{r_{i m, N+1}+r_{i m, N}} \\
& -\delta_{i} \frac{\phi\left(r_{i n, N+1}\right)-\phi\left(r_{i n, N}\right)}{r_{i m, N+1}-r_{i n, N}} \cdot \frac{z_{i, N+1}+z_{i, N}-z_{n, N+1}-z_{n, N}}{r_{i n, N+1}+r_{i n, N}} \\
& -g_{i}
\end{aligned}
$$

Difference equations (4.7)-(4.12) are consistent with differential equations (4.1)(4.6) and conserve exactly the same system invariants ${ }^{2,3}$ as do (3.1)-(3.3). System (4.7)(4.12), for each of $N=0,1,2,3, \ldots$, consists of 30 equations in the unknowns $x_{i, N+1}$, $y_{1, N+1}, z_{i, N+1}, v_{i, x, N+1}, v_{i, y, N+1}, v_{i, z, N+1}, i=1,2,3,4,5$; and in the knowns $x_{i, N}, y_{i, N}, z_{i, N}, v_{i, x, N}, v_{i, y, N}, v_{i, z, N}$. These are solved readily by Newton's method ${ }^{2}$.

In all the examples to be described in the next section, the results will be given relative to $P_{1}$, whose position and velocity coordinates at the end of each time step are set to $\overrightarrow{0}$. By this means, the motion of the hexahedron's geometric center $\bar{P}$ fully characterizes the motion of the gyroscope.

Typical FORTRAN programs for generating initial data and for trajectory calculation from given initial data are available for the interested reader in the Appendices of Greenspan 4 .

5. Examples. In considering examples, we must first choose a potential function $\phi$. We do this in cgs units and in such a fashion that we ensure that the tetrahedron is rigid. To accomplish this, we introduce the following classical, molecular type function ${ }^{5,6}$ :

$$
\phi=A\left[-\frac{1}{r_{i j}{ }^{3}}+\frac{1}{r_{i j}{ }^{3}}\right], A>0,
$$

in which $A$ is sufficiently large to impose rigidity. The choice of exponents in (5.1) prevents the explosive behavior characteristic of real molecules, yet allows for attractive and repulsive interaction, as is desirable for aggregates of molecules ${ }^{6}$. 

satisfies

From (5.1), it follows that the magnitude $F$ of the force $\vec{F}$ determined by $\phi$

$$
F=A\left[-\frac{3}{r_{i j}{ }^{4}}+\frac{5}{r_{i j}{ }^{6}}\right] \text {. }
$$

Thus, $F(\bar{r})=0$ provided $\bar{r}=(5 / 3)^{\frac{1}{2}} \sim 1.290994449$.

We now choose the parameter $R$ to be

$$
R=1.290994449 .
$$

For this value of $R$ the force between any two neighboring particles is zero, so that the gyroscope is physically stable. The parameters $A, g$, and $m_{i}$ are scaled for computational convenience to be $A=10^{6}, g=0.980, m_{i}=1, i=1,2,3,4,5$, unless otherwise indicated. The time step $\Delta t$ is chosen to be $\Delta t=10^{-5}$. In the figures to be given, units on the $Z$ axis are often rescaled to accentuate the character of the resulting trajectory. In all the calculations, the distance between any two neighbors is always 1.291.

In this first example, let $V=20, \alpha=8^{\circ}$. Figure 4 shows the resulting circular precession of the geometric center every 10000 time steps through 28000000 time steps. The graph consists of 2800 points. $\bar{P}$ has completed just over one complete rotation. the $\bar{z}$ values vary in the narrow range $1.0435<\bar{z}<1.04395$. Figure 5 shows the projection in the $X Y$ plane of the motion shown in Figure 4.

In the next six examples, $V=15$, but $\alpha$ varies successively through the values $15^{\circ}, 30^{\circ}, 45^{\circ}, 60^{\circ}, 75^{\circ}, 90^{\circ}$. The results are shown in Figures $6-11$. The figures reveal precession of the geometric center with the radius of the trajectory increasing with $\alpha$. For $\alpha=90^{\circ}$, one finds $-0.006<\bar{z}<0.000$.

For the next example, set $V=3, \alpha=16^{\circ}$. The resulting motion of the geometric center is the nutation shown in Figure 12. In this example $\bar{z}$ varies in the range $0.4<\bar{z}<0.54$, so that the gyroscope displays a periodic rising and falling motion. The projection of the trajectory in the $X Y$ plane is shown in Figure 13. Figures 12 and 13 indicate clearly the formation of cusps.

For our final example, choose again $V=15$ and $\alpha=15^{\circ}$, but let the mass $m_{2}$ of $P_{2}$ be 3 . Thus, the resulting hexahedron, though symmetric, is nonhomogeneous. The resulting trajectory of $\bar{P}$ is shown every 10000 time steps through 30000000 time steps in Figure 14. $\bar{P}$ has completed just over one complete rotation in this time and the graph contains 3000 points. That this graph is different from all previous graphs is quite visible. To analyze the resulting behavior in greater detail, we first examined the projection of the trajectory in the $X Y$ plane, since the variation in $\bar{z}$ was entirely in the small range $1.0155<\bar{z}<1.0205$. This projection is shown in Figure 15 and reveals that the variations of $X$ and $Y$ must be relatively complex. To study these variations more precisely, the motion was simulated over its first 500000 steps and plotted every 500 time steps. The projection of the resulting 1000 points in the $X Y$ plane is shown in Figure 16. What appears to be happening is that the $X$ and $Y$ coordinates are looping. However, plotting every 500 points has resulted in a graph with polygonal shapes rather than smooth shapes. To analyze the motion further, we concentrated only on the lower right corner of 
the graph show in Figure 16. For this purpose, we reran the trajectory but for only 150000 time steps and plotted every 50 time steps. The projected motion in the $X Y$ plane is shown in Figure 17 and contains 3000 points. What is revealed is both looping and cusp formation, which to us was totally unexpected.

6. Remarks. It would be most interesting to plot the trajectories shown in figures 14 and 15 with the accuracy used in Figure 17. However, to plot every 50 time steps through 30000000 time steps would require the ability to plot the resulting 600000 points. Our Delta Graph package did not have this capability. Further, one must be able to plot on a relatively large set of axes to exhibit the details of Figure 17 throughout the entire trajectory.

Though small perturbations of the parameters used for the results shown in Figures 4-13 resulted in entirely analogous results, the introduction of nonhomogeneity results in complexities which were unexpected. Though the motion of homogeneous, continuous gyroscopes can at times be analyzed mathematically, this does not seem to be the case when nonhomogeneity is introduced ${ }^{7,8}$. Further study of examples like the final one in Section 5 are in progress, but the graphics difficulties and the large number of parameter choices under study are resulting in very slow progress.

Finally, note that any other classical molecular type function which ensures rigidity can be used in place of (5.1). 
$08=0 \cdot 0 Z=\Lambda$ is e $2 \pi 8 \mathrm{IJ}$

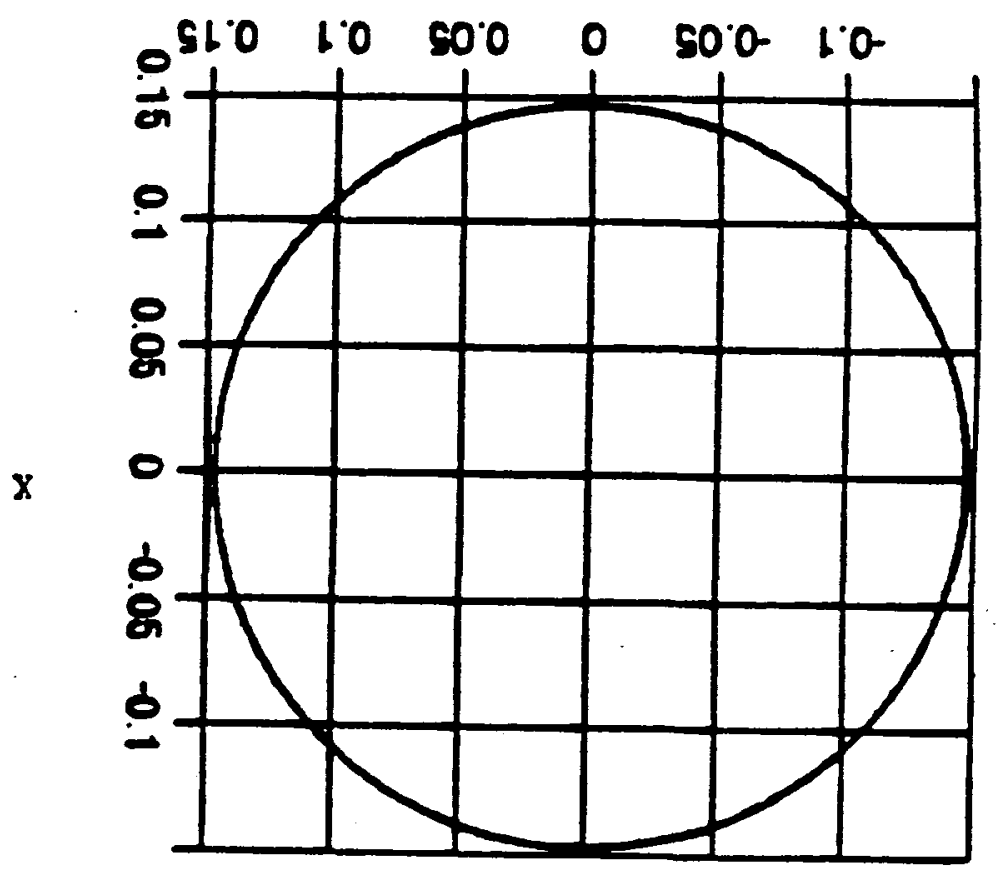

$.8=\varnothing \quad 02=\Lambda \quad t \quad 2 \pi 8 \mathrm{IJ}$

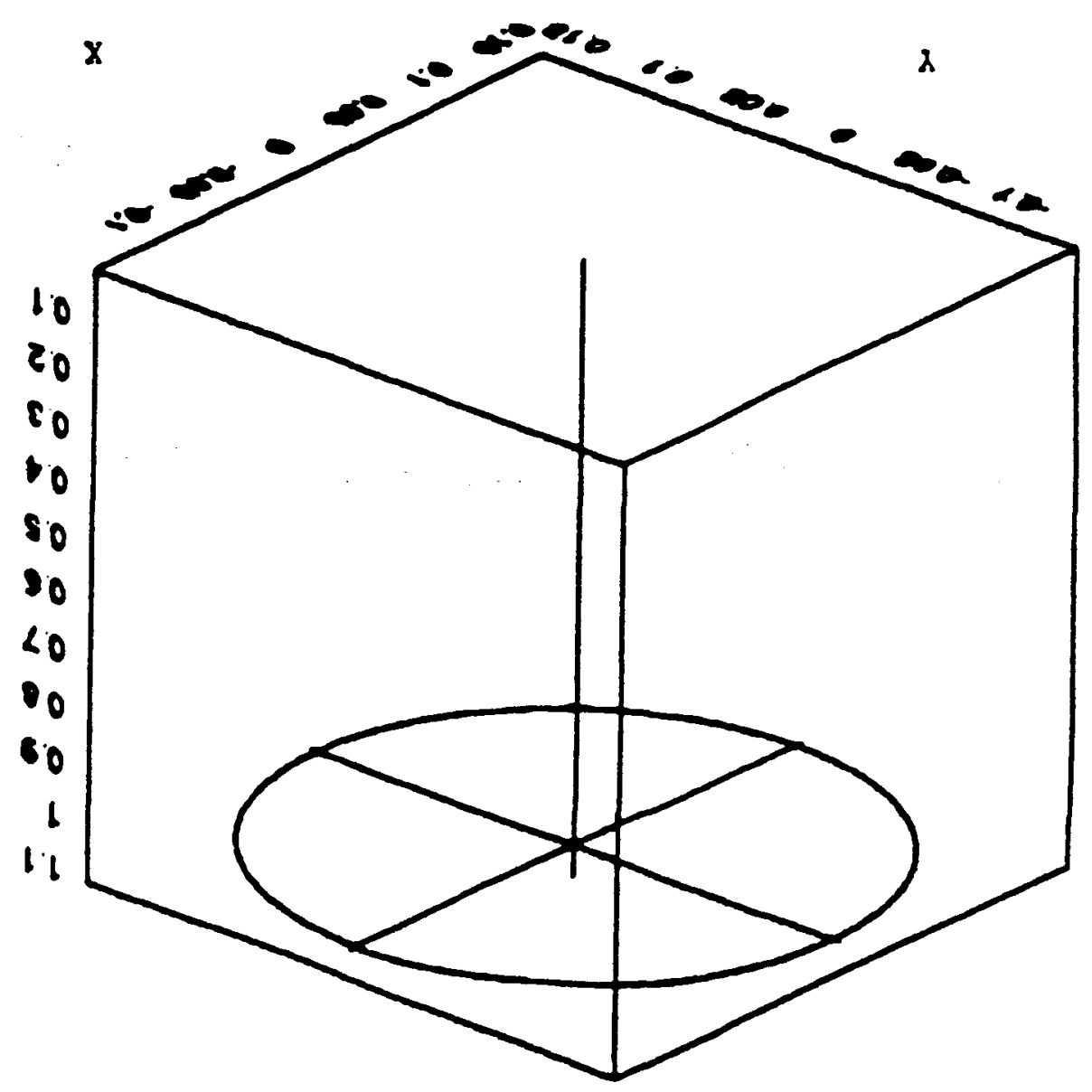



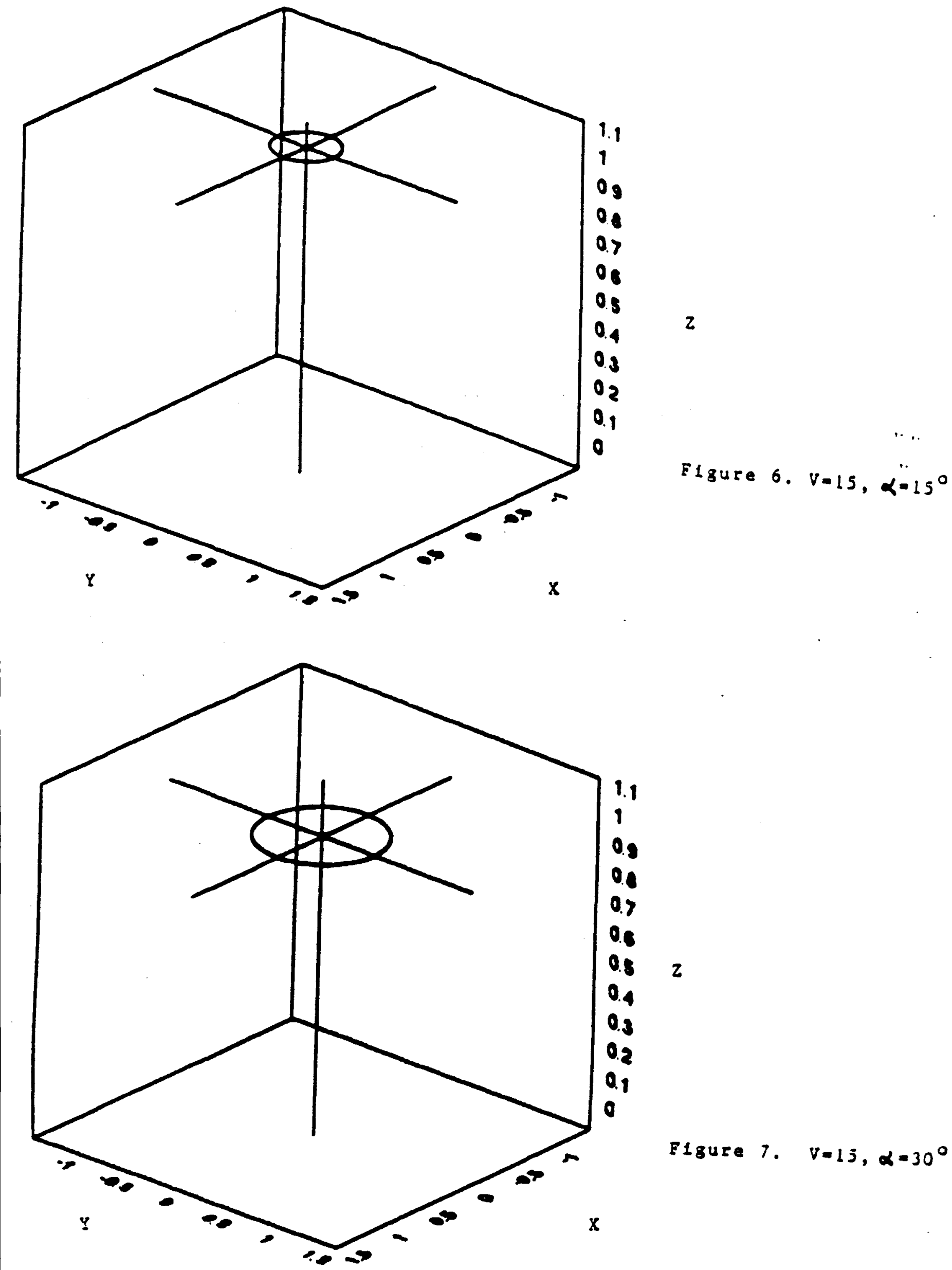
$009=P \quad S T=\Lambda \cdot 6$ ens II

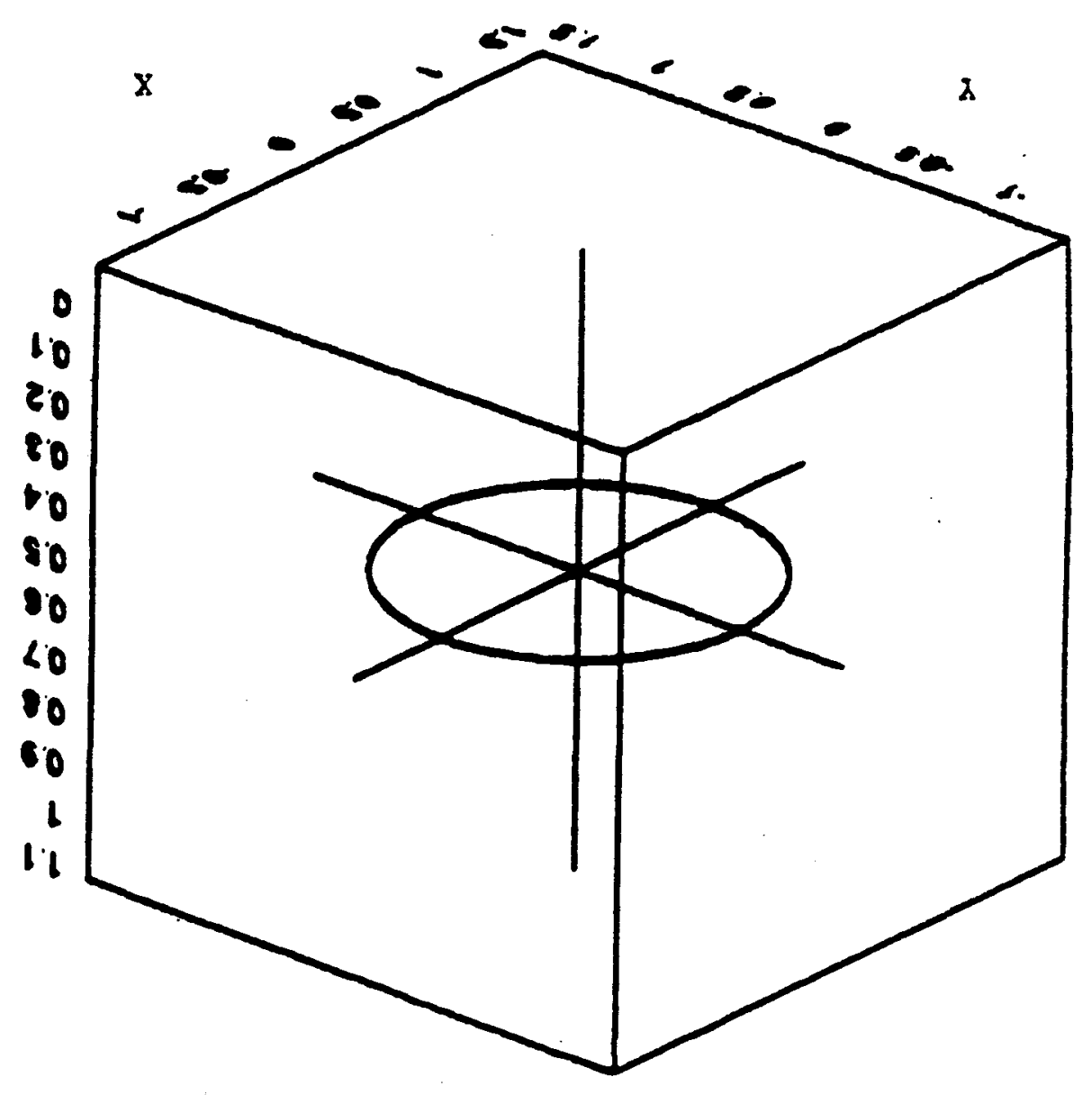

$057=9 \cdot 51=1 \cdot 8$ e $2 \pi 8 T a$

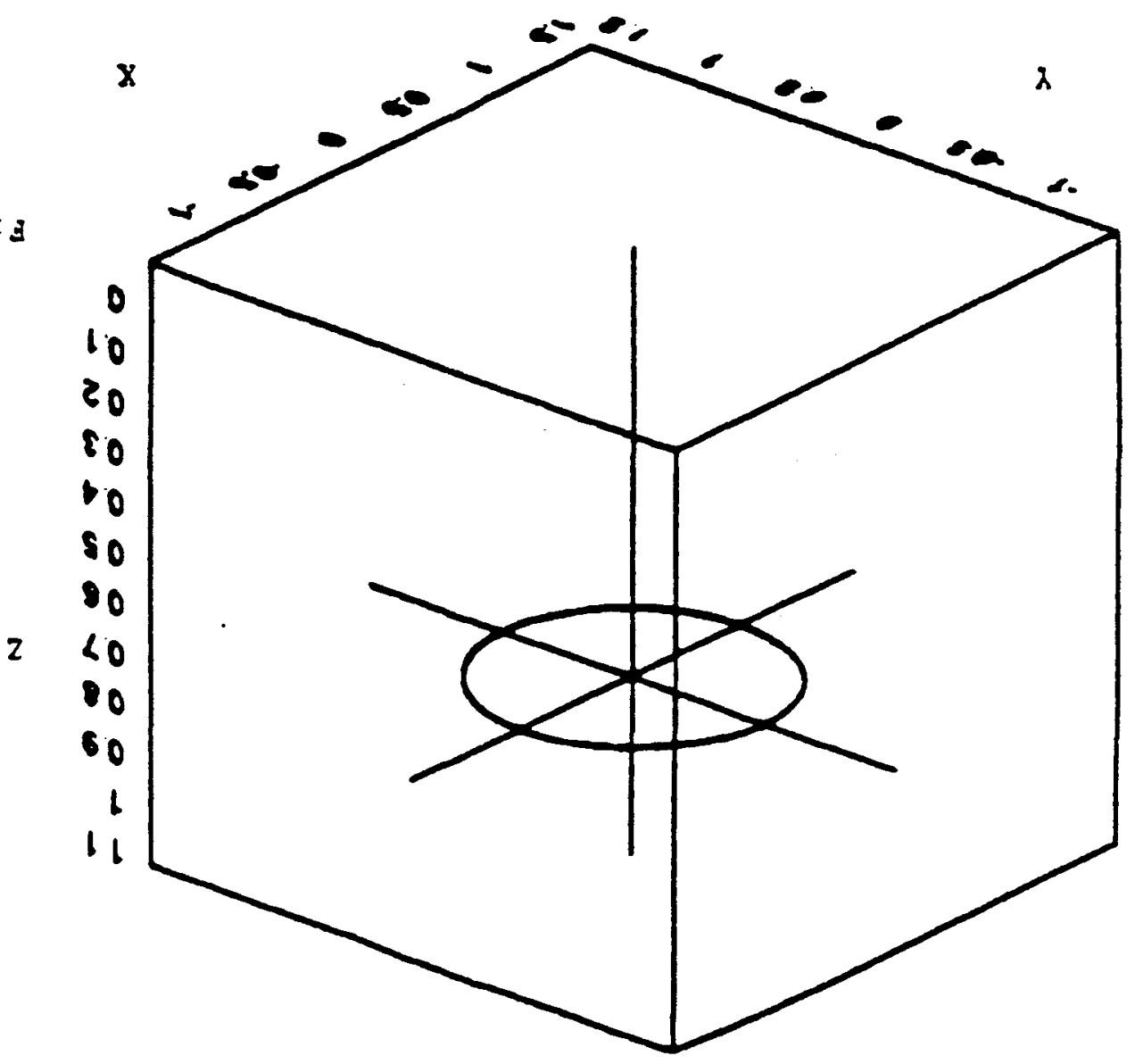



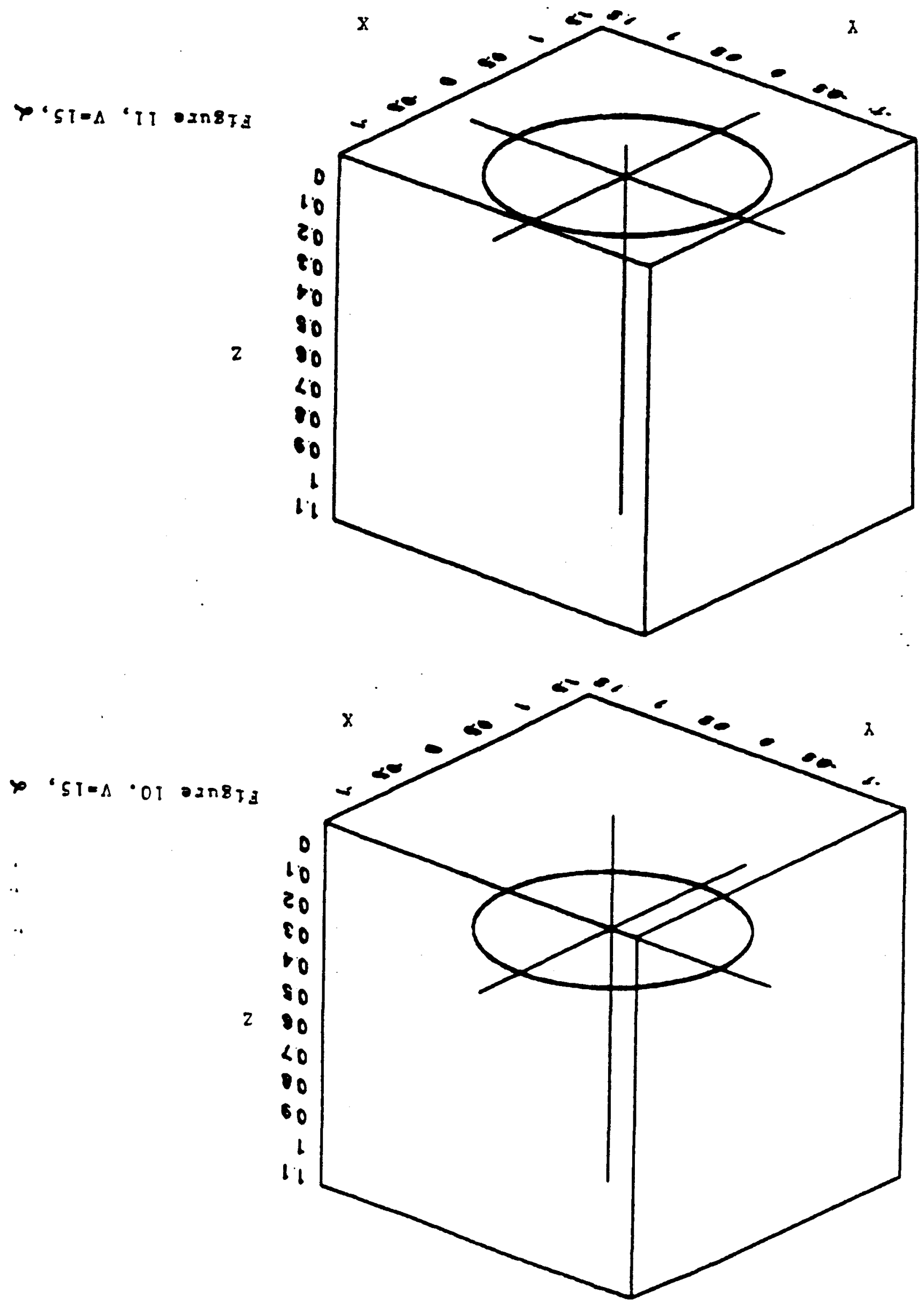
$\lambda$

$091=7 \cdot \varepsilon=\Lambda \cdot \varepsilon \tau \cdot \operatorname{sn} 8 \mathrm{~F}]$

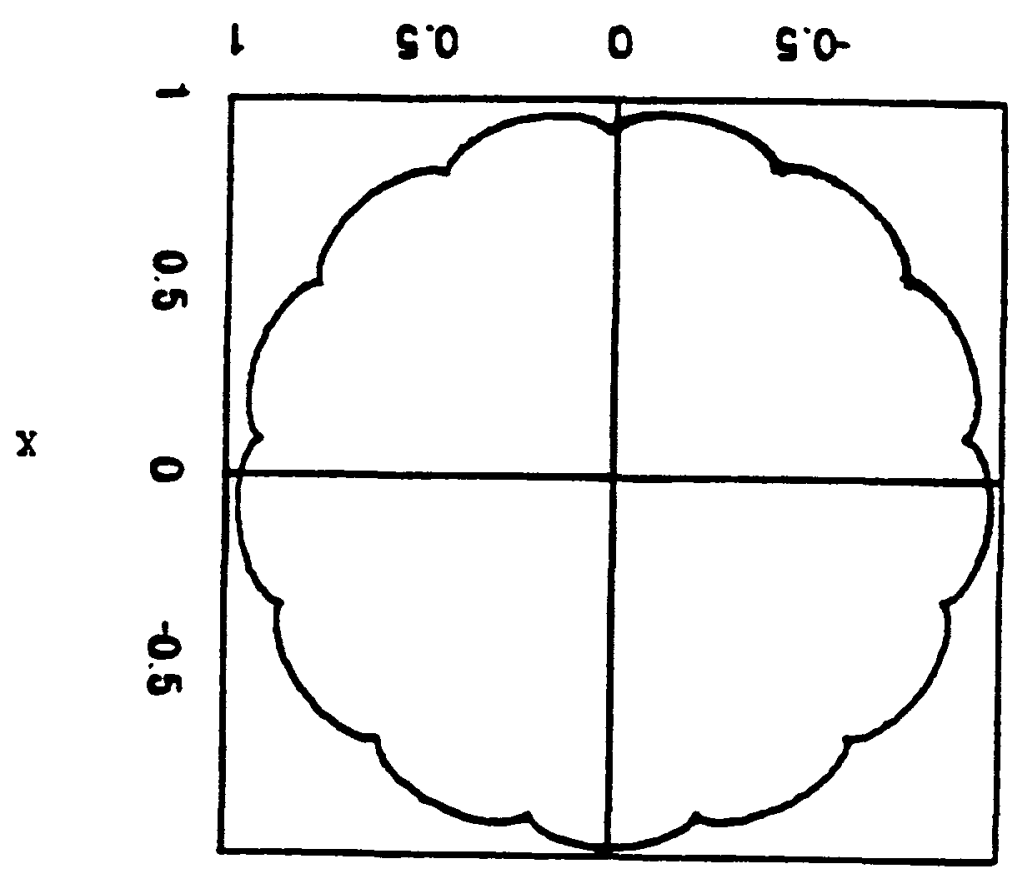

$091=0 \cdot c=1 \cdot 21$ - $2 \pi 813$

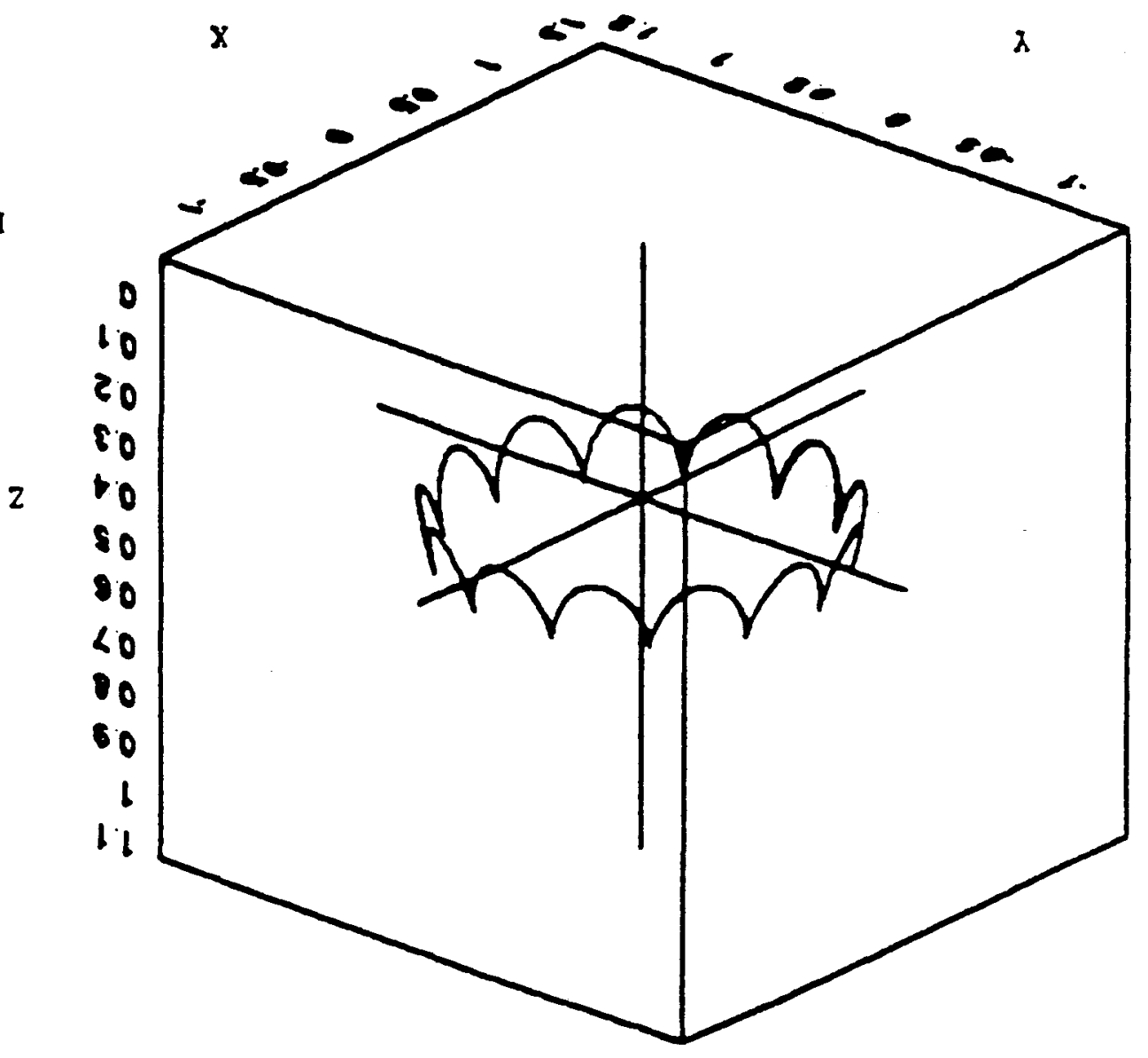




$$
\frac{9}{0}
$$




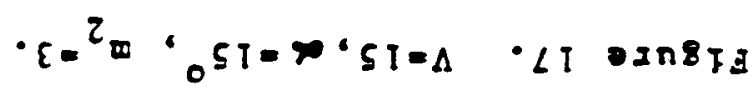
$x$

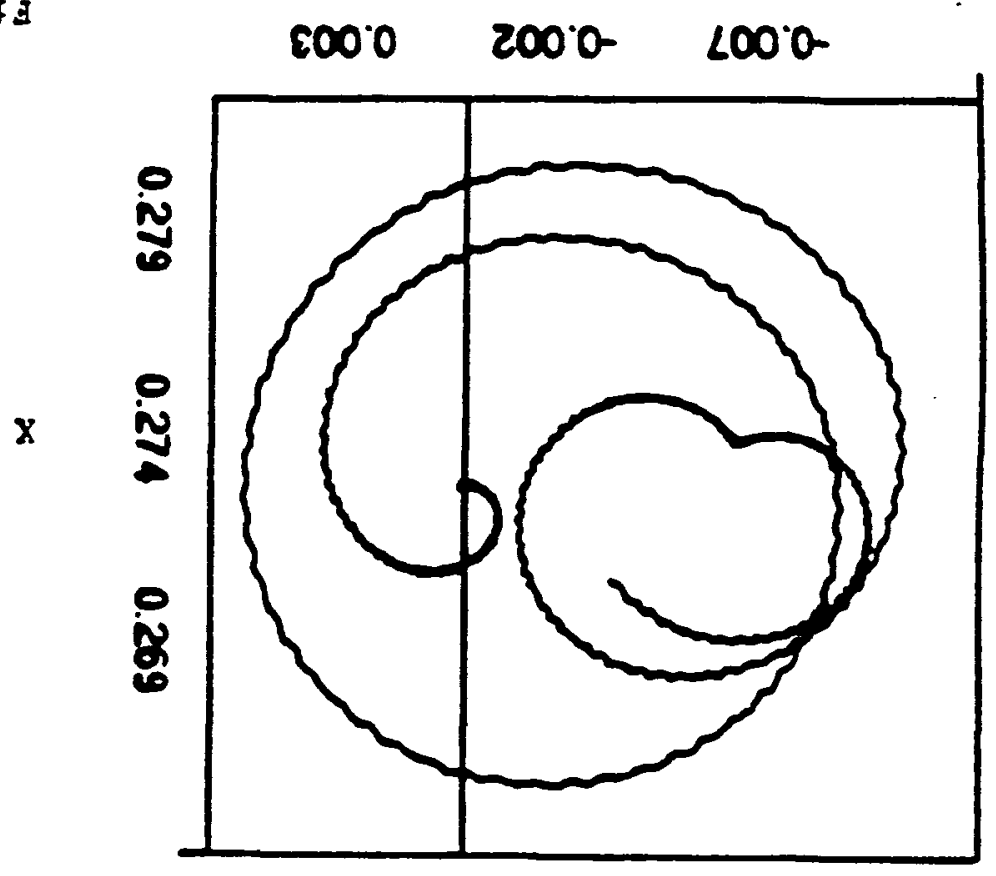

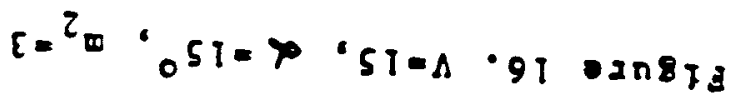

$\lambda$

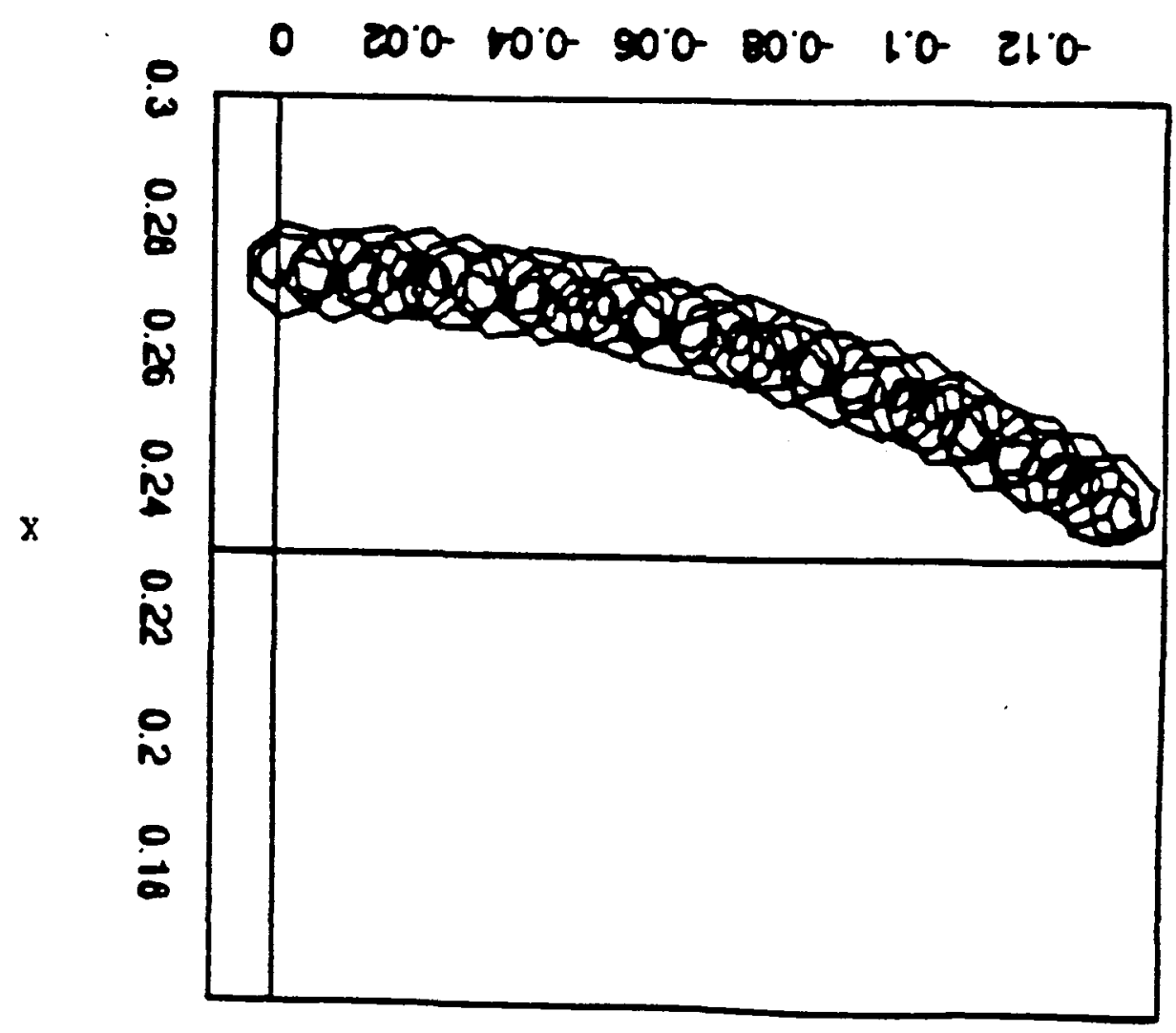




\section{REFERENCES}

1. H. Goldstine, CLASSICAL MECHANICS, 2nd Edition, Addison-Wesley, Reading, 1980, Chaps. iv, v.

2. D. Geenspan, ARITHMETIC APPLIED MATHEMATICS, Pergamon, Oxford, 1980.

3. D. Greenspan, "Completely Conservative, Covariant Numerical Methodology", Comp. Math. Applic., 29, 1995, p. 37.

4. D. Greenspan, IR 315, Math. Dept., UT Arlington, Arlington, Texas, 1996.

5. R. P. Feynman, R. B. Leighton, and M. Sands, THE FEYNMAN LECTURES ON PHYSICS, vol. I, Addison-Wesley, Reading, 1963.

6. D. Greenspan, QUASIMOLECULAR MODELIING, World Sci. Press, Singapore, 1991.

7. L. D. Landau and E. M. Lifshitz, MECHANICS (3rd Edition), Pergamon, Oxford, 1976.

8. A. Gray, GYROSTATICS AND ROTATIONAL MOTION, Dover, N.Y., 1959. 
PRIME SOCROINATES.

$X ! P=\langle 1$-COS $(A L P H A)+Z 1 * 5 I N(A L=H A$;

$Y|P=Y|$

$\because ! P=-X \mid-S I N(A L P H A)+? 1=C O S(A L=H A$;

$\times 2 ?=X ?=\operatorname{COS}(A L P H A)+Z ? \times S ? V(A L=+A)$

$\because 20=Y$ ?

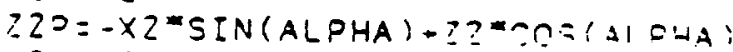

$\times 3 \circ=\times 3^{*} \operatorname{COS}(A L O+A)+? 3^{-}=T N(A L P H A)$

$Y 20=\cdot 3$

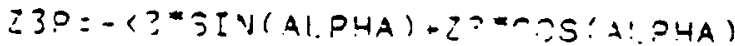

$\because ' P=X^{\prime}=O S S(A L P H A)+Z \div=5: \because(A L P H A)$

$\because 1 P=Y 4$

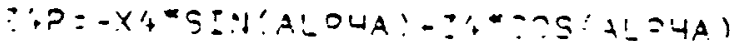

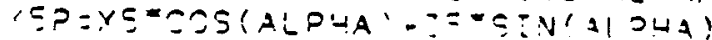

$\because 5 ?=y 5$

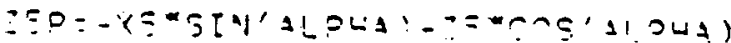

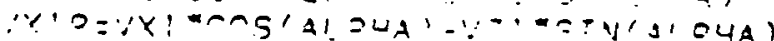

$\because, P=\because, y$

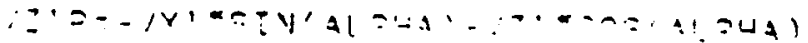

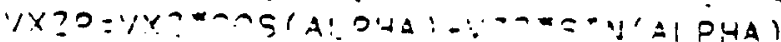

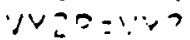

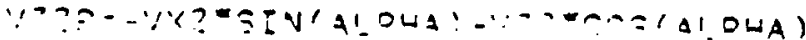

$\because \times ?=: \because \times ?$ ?

$\because \cdots P-\because 13$

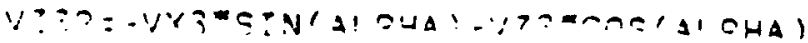

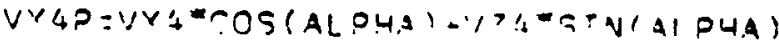

भ's $P=V / 4$

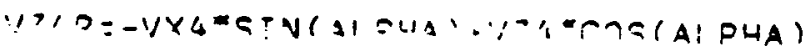

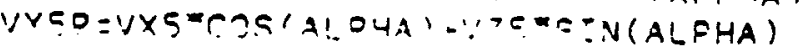
VYSO =yYS

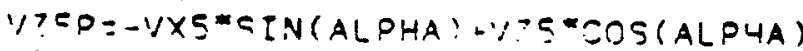

$Y !=X I P$

$Y:=Y \cdot P$

$?:=? ! P$

$\times 2=\times 2 P$

$\checkmark T=Y 2 P$

$\geq ?=? ? 0$

$\times 3=\times 3 P$

$Y 3=Y 3 P$

$23=23 P$

$X 4=X 4 P$

$Y 4=Y 4 P$

$? 4=? 4 P$

$Y 5=X 5 P$

$Y 5=Y 5 P$

$25=25 P$

XSARNEW $=(X 1+X 2+X 3+X 4+X 5) / 5$.

YQARNEW $=(Y 1+Y Z+Y 3+Y 4+Y 5) / 5$.

Z3A.PNEW $=(21-22+23+24+25) / 5$.

$V X|=V X| P$

$V Y 1=V Y 1 P$

$V Z 1=V Z 1 P$

Y $\times 2=V \times 2 ?$

$\because Y Z=V \vee ? \circ$

$V Z Z=V Z 2 P$

$V \times 3=V \times 30$

VY $3=V Y 3 P$

$V 23=V 23 ?$

$\because X_{4}=1 / X_{4} P$

$V Y S=1 Y 40$ 


3กNILNOS

(I) $02 A=\left(1^{\circ}\right.$ I) $2 A$

(I) $O A A=\left(1^{\circ}\right.$ I) $\mathrm{AN}$

(I) OXA $=\left(1^{\circ}:\right) \times A$

(I) $O Z=\left(i^{\cdot} i\right) z$

(I) $O A=: 1 \cdot I$ in

(I) $0 x=(1 \cdot I) X$

$S \cdot 1=I$ or OC

-

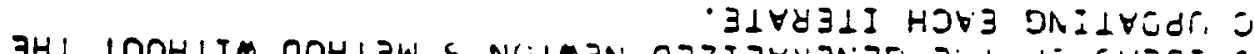

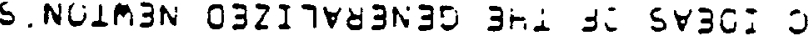

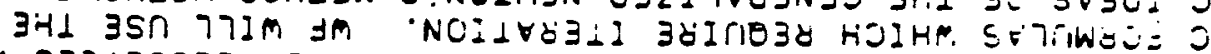

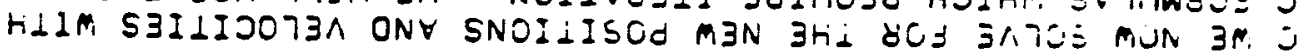

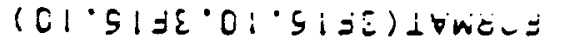

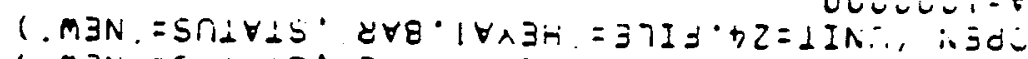

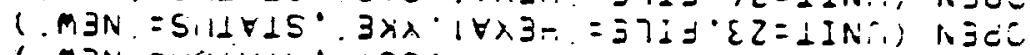

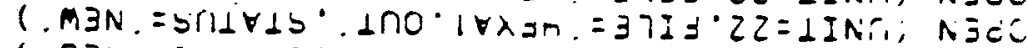

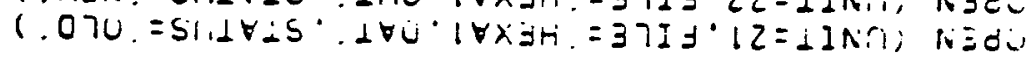

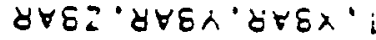

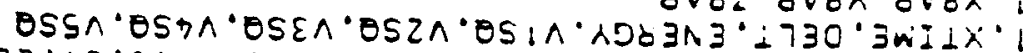

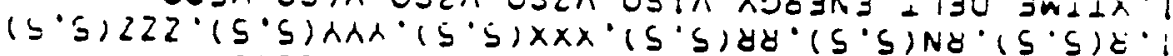

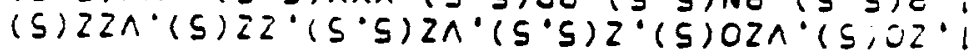
( S)AAn (S) $\times \times A \cdot(S) \lambda A \cdot(5) \lambda x$ :

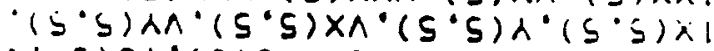

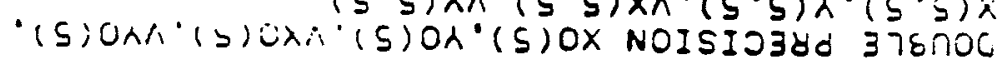

yt 7ח5N OWNLN3WOW a

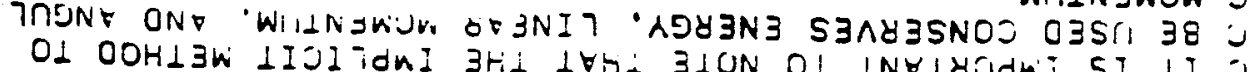




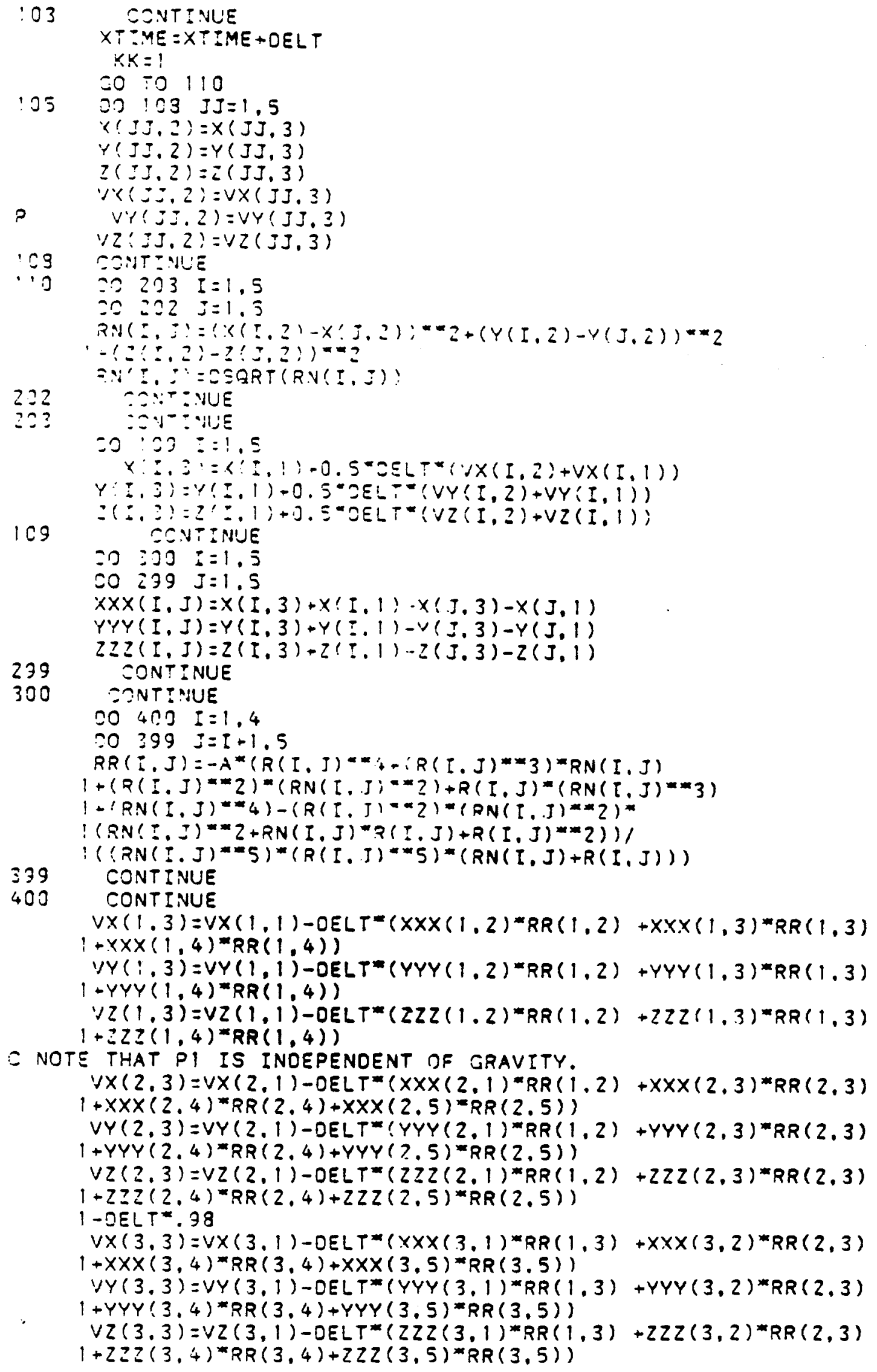


$1-D E L T * .98$

$V X(4,3)=V X(4,1)-C E ! T=(X X X(4.1) \times P R(1,6)+Y X X(4,2) * R R(2,4)$

$1+X X X(4,3) * R R(3,4)+X X X(4,5) * F Q(4,5))$

YY'i, 3) $=V Y(4,1)-D E L T=(Y Y Y(4.1)=R R(1.4 !+Y Y Y(4.2)=R R(2.4)$

1 -YYY (4.3) $=R R(3.4)+Y Y Y(4.5)=R R(4.5)$ !

$Y Z(1, .3)=V Z(4.1)-D E L T=(Z Z ?(4.1:=8 R(1.4)+7-Z(4.2) * R R(2.4)$

$1+Z Z Z(4.3)=R R(3.4)+Z Z Z(4.5) * R R(4.5)$

$1-3 E L T-98$

$\because Y(5.3)=V Y(9.1)-D E L T=(X Y X(5.2)=R R(2.5)$

' XXX:5. 3) $R R(3.5 !+Y X X(5.4)=R R(4.5))$

WY!S. 3) =WY (5.1)-กFLT* (YYY (5.2) $=R R(2.5)$

' UYV: 5.3) $R R(3.5)+Y Y Y(5.4)=R R(4.5))$

$\because \geq(5.3)=V Z(5.1)-D E L T=(Z Z Z(5.2)=R R(2.5)$

$1-Z Z Z(5.3) * R R(3.5)+2 Z 2(5.4)=R R(4.5))$

- $-0 E L T=.98$

$<K=K K+1$

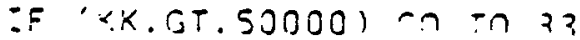

ก $9975 \quad I=1.5$

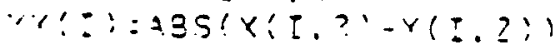

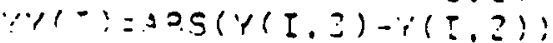

$\because Z I:=13 S(z(I, 3)-Z(I, Z))$

$\because Y X ! Z)=A B S(\forall x([.3)-\because x ![.2))$

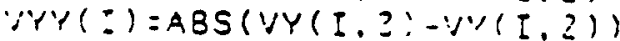

3875

$\forall Z Z(!)=A 3 S(V Z(I, 3)-V Z([, 2))$

:NCEX $=-1$

כก $500 \quad I=1.5$

IF (XX(I).GT.0.000200000005) INOEX $=+1$

IF (YY(I).GT.0.0OOCONJOCOOS) INDEX $=+1$

IF (ZZ(I).GT.0.000000000005) INDEX $=+$ :

IF $(V X X(I) . G T .0 .000000000005)$ INOEX $=+1$

IF (VYY(I).GT.0.000000000005) INDEX $=+1$

600

IF (VZZ(I).GT.0.003000000005) INOEX $=+1$ CONTTNUE

IF (:NOEX.EQ.+1) GO TO 105

J0 $1008 \quad J J=1.5$

$x(J . J .2)=x(J J, 3)$

$Y(J J, 5)=Y(J J, 3)$

$Z(J J, Z)=Z(J J, 3)$

$V X(J J .5)=V X(J J .3)$

$\because Y(J J, Z)=V Y(J J, 3)$

$V Z(J J, Z)=V Z(J J, 3)$

1009 CONTINUE

C WE YNW DETERMINE OATA RELATEVE TO $P 1$.

$Y(2,2)=X(2,2)-X(1,2)$

$Y(2,2)=Y(2,2)-Y(1,2)$

$z(2,2)=z(2,2)-z(1,2)$

$X(3.2)=X(3.2)-X(1,2)$

$Y(3,2)=Y(3,2)-Y(1,2)$

$z(3.2)=2(3.2)-2(1.2)$

$X(4.2)=X(4.2)-X(1,2)$

$Y(4.2)=Y(4,2)-Y(1,2)$

$2(4,2)=2(4,2)-2(1,2)$

$X(5,2)=X(5,2)-X(1,2)$

$Y(5,2)=Y(5,2)-Y(1,2)$

$z(5,2)=z(5,2)-2(1.2)$

$X(1.2)=0.0$

$Y(1.2)=0.0$

$?(!, 2)=0$.

$v x(2.2)=v \times(2.2)-v \times(1.2)$ 


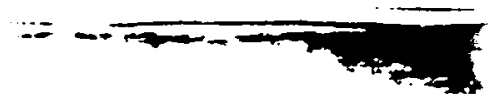

$V Y(Z . Z)=V Y(2.2)-V Y(1.2)$

$V Z(2.2)=V Z(2,2)-V Z(1,2)$

$V X(3,2)=V X(3,2)-V X(1,2)$

$\operatorname{VY}(3,2)=V Y(3,2)-V Y(1,2)$

$V Z(3,2)=V Z(3,2)-V Z(1,2)$

$V \times(4.2)=V X(4,2)-V X(1,2)$

$V Y(4,2)=V Y(4.2)-V Y(1.2)$

$V I(4.2)=V Z(4,2)-V Z(1.2)$

$V X(5,2)=V X(5.2)-V X(1.2)$

$V Y(5.2)=V Y(5.2)-V Y ! 1.2)$

$V Z(5.2)=V Z(5.2)-V Z(1.2)$

$V \times(1.2)=0.0$

भY $(1,2)=0.0$

$\forall \geq(1, ?)=?$.

$k=x+1$

If 'MOD(K.KPRINT). T. O) r:o $\div 082$

V' $3=V Y(1.2) \cdots V X(1.21+V Y(1.2)-V Y(1,2)+V Z(1,2)=V Z(1,2)$

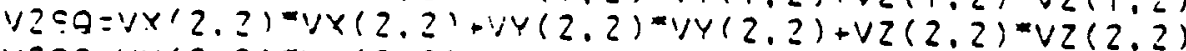

VЗSQ $=V Y(3,2) \neq V Y(3.2)+Y Y(3.2)-V Y(3,2)+V Z(3,2)-V Z(3.2)$

$V 45 Q=V Y(4.2)=V X(4.2)+V Y(4.2)-V Y(4.2)+V Z(4.2)=V Z(4.2)$

$V 55 \theta=V \times(5.2)-1 / X(5.2)+V Y(5.2) * V Y(5.2)+V Z(5.2)-V Z(5.2)$

ENERSY $=-A /\left(\left(R\left(! .2^{1-3}-3\right)\right)-A /((R(1,3)=3))\right.$

$!-A /((R(1.4)=-3))-A /((R(2,3)=3))$

$!-A /((R(2.4)=-3))-A /((R(2.5)=-3))$

$!-A !((R(3.4)=3))-A /((R(3.5)=-3))$

$1-A /((R(4.5)=3))$

$1+A /((R(1,2)==5))+A /((R(1.3)=-5))$

$1+A,((R(1,4)=5))+A /(\{R(2,3)=-5))$

$1+A /((R(2,4)=5))+A /((R(2,5)=5))+A /((R(3,4)=5))$

$1+A /((R(3,5)-5))+A /((R(4,5)=5))$

$1+.98=(Z(2, Z)+Z(3,2)+Z(4,2)+Z(5,2))$

ENERGY = ENERGY + (VISQ $+V 2 S Q+V 3 S Q+V 4 S Q+V 5 S Q)$

ENERGY $=.000001^{-} E N E R G Y$

WRITE $(23,8000) \mathrm{K}$, ENERGY, RN $(1,2), \operatorname{RN}(1,3), R N(1,4)$,

8000

$\operatorname{IRN}(2,3), \operatorname{RN}(2,4), \operatorname{RN}(3,4), \operatorname{RN}(2,5), \operatorname{RN}(3,5), \operatorname{RN}(4,5), \dot{X T I M E}$ FDRMAT (II3.F12.5.9F 7.3.F12.5)

$X B A R=(X(1,2)+X(2,2)+X(3,2)+X(4,2)+X(5,2)) / 5$.

YSAR $=(Y(1,2)+Y(2,2)+Y(3,2)+Y(4,2)+Y(5,2)) / 5$.

$Z B A R=(2(1,2)+Z(2,2)+Z(3,2)+Z(4,2)+Z(5,2)) / 5$.

C NOTE THAT XBAR, YBAR. ZBAR ARE RELATIVE TO DI SO THAT

C WE CAN EASILY PLOT THE MOTION OF THE MASS C.ENTER.

WRITE $(24,938)$ XBAN, YMA, $28 A R$

939 FORMAT (3F16.10)

82

9383

IF (K.LE.10000000) 60 TO 65

WRITE $(22,10)(X(I, Z), Y(I, Z), Z(I, Z), V X(I, 2)$.

$\operatorname{IVY}(I .2), V Z(I . Z), 2 Z 1,5)$

8050

WRITE $(22.8050)$ XTIME

83

FORMAT (F|5.5)

END 